\title{
Propuesta de mejoramiento del Impuesto a las personas en Chile
}

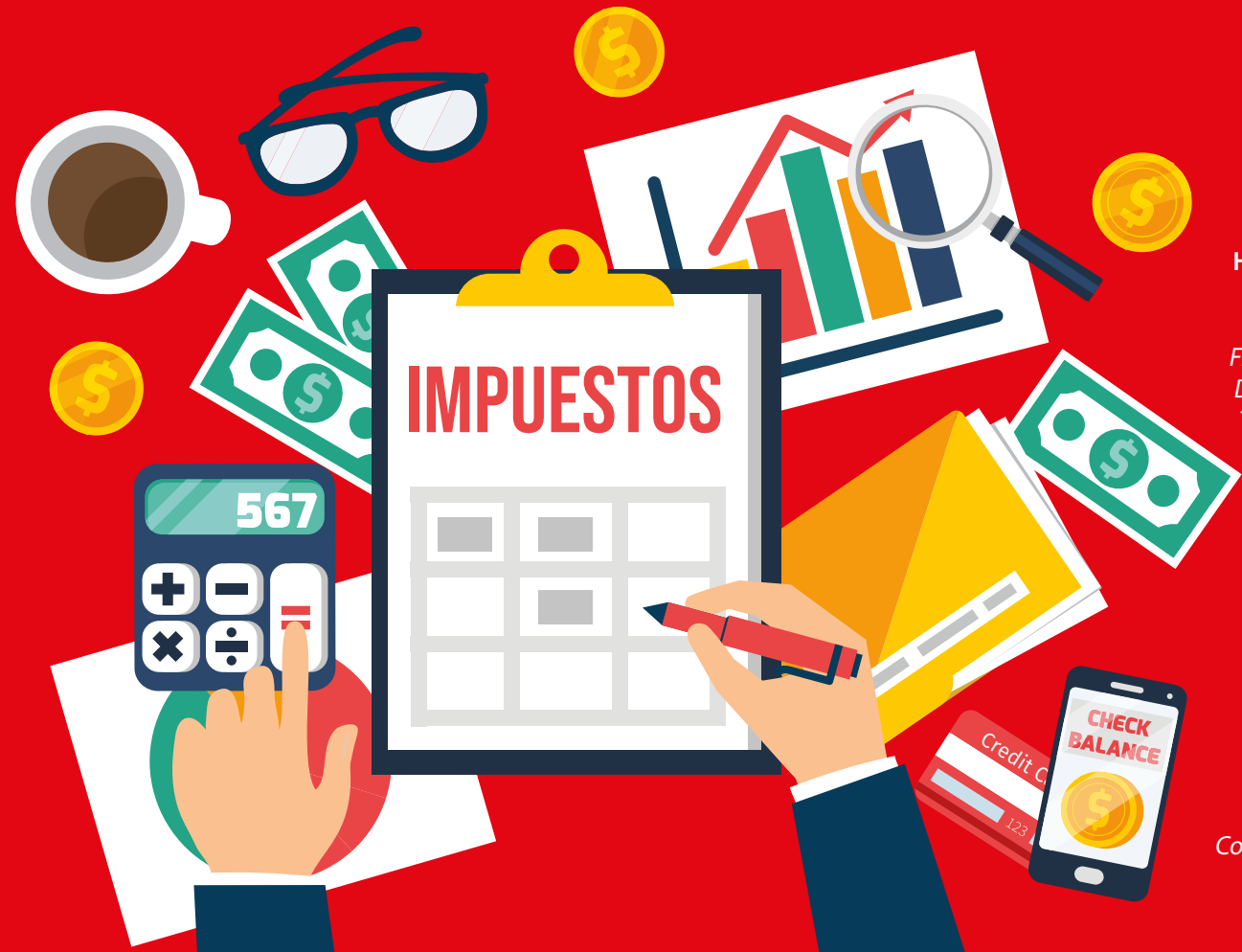

Humberto A. Borges Quintanilla Académico FEN-UAH

Doctor $\odot$ en Contabilidady Finanzas, Universidad de Zaragoza Director del Diplomado en Gestión Tributaria e investigador asociado al Centro Interdisciplinar de Políticas Públicas CiPP

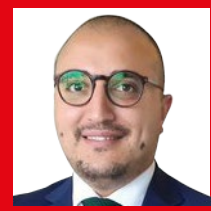

Nelson Valenzuela Magister en Derecho Tributario, Pontificia Universidad Católica Consultor tributario independiente y Docente FEN U. de Chile

Este articulo analiza los efectos redistributivos de los impuestos en Chile y plantea que el Impuesto Global Complementario (IGC) podría recaudar más de las regiones con mayores ingresos. Se propone aumentar el IGC, principalmente en el grupo de contribuyentes de mayores ingresos que fue beneficiado con la política de disminución de impuestos aplicada en 2014.

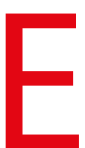

$\mathrm{n}$ los tiempos que vive Chile, debemos revisar las políticas tributarias, por cuanto estas tienen impacto directo en la recaudación fiscal que da origen a los programas sociales impulsados por cada Gobierno. En especial, en esta oportunidad, quisiéramos centrar nuestro análisis en el Impuesto a las Personas Residentes, las tasas aplicables en cada tramo, sus beneficios y rebajas que establece la norma para estos contribuyentes, los cuales en nuestro país se afectan con el Impuesto Global Complementario (IGC).

Las razones para revisar los impuestos a las personas físicas residentes (IGC), se basan en que este gravamen ha sido cuestionado por la OCDE en términos de su real impacto en la recaudación fiscal total, la misma que recae principalmente en el Impuesto al Valor Agregado (IVA), un tributo a todas luces de carácter regresivo.

Adicionalmente, podemos indicar que la recaudación por impuesto a las personas en Chile es inferior a la recaudación de los impuestos progresivos de otros países miembros de la OCDE. Además, los cambios introducidos por la reforma tributaria aprobada en el año 2014 han provocado mayores distorsiones, destruyendo la condición progresiva del IGC y produciendo un deterioro de la distribución equitativa referidas a los tramos impositivos y regio- nales (Borges, 2019). Sin perjuicio de ello, el cambio legislativo del 2020 impulsó un giro en la política tributaria, apuntando a una mayor recaudación, reponiendo el tramo más alto de la tabla de tributos del IGC, pero no es suficiente.

Ahora bien, el estudio de las políticas impositivas debería centrar su análisis en el cuerpo normativo y en los efectos económicos que conlleva la estructura tributaria específica de un país $y$, en particular, observando con detención la capacidad redistributiva de la riqueza que permite dicho sistema, mediante el estudio de los efectos distributivos totales de todos los impuestos. 
En Chile, con el llamado"Estallido Social de octubre del 2019", muchas de las discusiones políticas y sociales se centraron en la falta de políticas públicas distributivas de la riqueza y de acceso igualitario, ya sea centradas en la educación, salud o falta de oportunidades para lograr mejorar los ingresos de las familias.

\section{Generalidades OCDE}

En general, la fuente de recaudación más importante en la estructura tributaria para el informe OCDE del año 2019 corresponde a las contribuciones a la seguridad social con un $26 \%$, seguido por el impuesto sobre la renta de individuos con un $24 \%$, y en tercer lugar el IVA con un $20 \%$. Con una menor participación quedan otros impuestos al consumo con una participación del $13 \%$, impuestos a las empresas con $10 \%$ y todos los otros impuestos con 7\%. (OCDE, 2020) En cambio, la estructura tributaria de Chile corresponde a las contribuciones a la seguridad social con un $7 \%$, impuestos sobre la renta de individuos con un $7 \%$, IVA con un $40 \%$, otros impuestos al consumo con una participación del 13\%, impuestos a las empresas con $22 \%$ y todos los otros impuestos con 11\%. (OCDE, 2020)

La estructura tributaria chilena es todo lo opuesto de la estructura promedio de los países OCDE. En primera instancia, muchos de los países de la OCDE la importancia mayor la tienen los impuestos al ingreso de los individuos y las contribuciones a la seguridad social. En cambio, en Chile para estas fuentes se registra una recaudación de tributos en proporción más bajas del grupo de países en promedio de la OCDE. Además, las AFPs privatizan la seguridad
"En Chile, con el llamado 'Estallido Social de octubre del 2019', muchas de las discusiones políticas y sociales se centraron en la falta de políticas públicas distributivas de la riqueza y de acceso igualitario, ya sea centradas en la educación, salud o falta de oportunidades para lograr mejorar los ingresos de las familias"

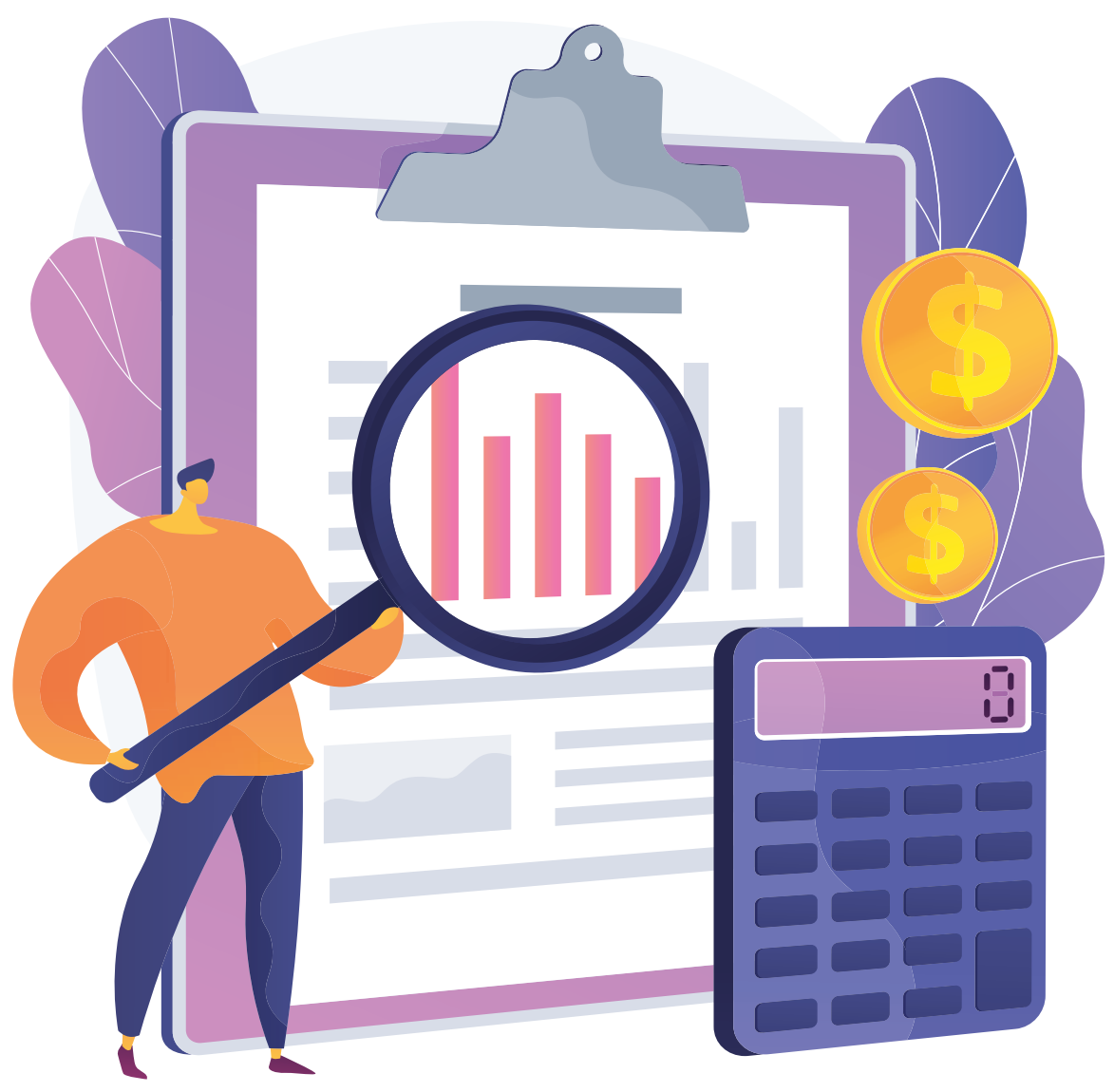

social enfocada en rentas de jubilación, que en otros países es parte de las fuentes recibidas por el Estado. Chile también tiene el porcentaje más alto de recaudación por concepto de IVA, con un $40 \%$ y por impuesto a la renta de las empresas con un $22 \%$, el segundo más alto después de México, con los obvios efectos que esta situación encubre, políticas tributarias regresivas que impactan fuertemente en la población de menores ingresos. Esto es respaldado por Jorratt y Martner (2020) en el documento Justicia Fiscal para Chile. Bajo este escenario, es indudable que el sistema de impuestos personales debe ser reformado para que en efecto pueda tener un carácter redistributivo y progresivo, logrando finalmente en términos de la carga tributaria un promedio cercano al que ostenta la OCDE para las personas físicas.

Sin perjuicio de lo anterior, y centrándonos en la creciente demanda social por más y mejores oportunidades de acceso a educación, salud y pensiones, es necesario visualizar una reformar a nuestro sistema tributario, para eliminar las distorsiones que hoy generan menos recaudación, exenciones que no tienen una justificación y reformular el impuesto a las personas.

\section{Efectos distributivos por tramo imponible y regional del IGC}

Debemos analizar el IGC desde los efectos distributivos totales, es decir, analizar la fracción de impuesto que se recauda $y$, conjuntamente, el efecto de los gastos sociales. La idea es analizar el componente referido a los impuestos recaudados y el uso de estos en los gastos que la sociedad requiere.

Respecto de los gastos sociales, la distribución de los recursos obtenidos por vía de impuestos debe ser focalizados, principalmente, en las regiones o zonas con mayores rangos de pobreza o con alguna debilidad estructural. Según De Zauzu (2012) una buena distribución de los recursos produce un efecto de bienestar social y aceptación de las instituciones políticas que gobiernan un país. Al no realizarse una buena distribución produce un efecto que se refleja en brechas sociales muy nocivas, las cuales corroen por dentro a la sociedad, por ejemplo, generando conflictos sociales en el tiempo (ver Perez \& Sandoval, 2020, Garretón, Joignant, Somma y Campos. COES, 2018). 
Análisis realizados con el PNUD (2018) "Desigualdad regional en Chile. Ingresos, salud y educación en perspectiva territorial", mostró brechas enormes entre las regiones en las dimensiones de Salud, Ingreso y Educación. Al comparar los datos entregados por el informe del PNUD y los ingresos fiscales generados por IGC (Borges 20201 y 2019; Ver apéndice I).

\section{Política Tributaria y Desarrollo Humano Regional en Chile}

La política tributaria en Chile, no se encuentra unida a una política de desarrollo de país. Es más, se puede determinar que ha tenido efectos más bien centralistas y favoreciendo algunas industrias sobre otras. Respecto a la crítica y reflexión sobre el IGC y las regiones, podemos centrarnos en Borges (2019), donde podemos observar que existe una desigualdad territorial y, por tramos impositivos. Respecto al último punto, podemos observar que los tramos fueron cambiados durante el 2014 (Reforma Tributaria - Gobierno de Michelle Bachelet) y la actual reforma de enero 2020 (Modernización Tributaria - Gobierno de Sebastián Piñera). El Cuadro 1 muestra que un gran grupo de contribuyentes han sido beneficiados con una rebaja impositiva. Al observar el cuadro 1 podemos observar que el tramo de contribuyentes de 150 UTA a 310 UTA, continua con una exención de impuesto, la cual proponemos que debe ser repuesta con una tasa del 37,5\% (o recuperar el $40 \%$ aumentando a $43 \%$ el tramo de 310 UTA en adelante), con lo cual estas personas de ingreso alto, entregaran realmente un aporte mayor a las arcas fiscales, recordando que ellos fueron favorecidos por una disminución de tasa de $40 \%$ a 35\% des el 2015 a 2020. La reposición del tramo de Modernización Tributaria 2020, sólo repone una parte de los tributos que no se han cobrado.

Cuadro $\mathrm{N}^{\circ} \mathbf{1}$

Tasas IGC con cambio en los respectivos años más propuesta

\begin{tabular}{|c|c|c|c|c|}
\hline Tramo en UTA & Tasa IGC Antes & Tasa IGC 2015 & Tasa IGC 2021 & Propuesta \\
\hline 120 a 150 UTA & $37 \%$ & Remplazada & & \\
\hline más de 150 UTA & $40 \%$ & Eliminada & & \\
\hline más de 120 UTA & & $35 \%$ & Remplazada & \\
\hline de 120 a 310 UTA & & & $35 \%$ & Remplazada \\
\hline más de 310 UTA & & & $40 \%$ & Remplazado \\
\hline de 120 a 150 UTA & & & & $35 \%$ \\
\hline de 150 a 310 UTA & & & & $\mathbf{3 7 , 5 \%}$ a $\mathbf{4 0 \%}$ \\
\hline más de 310 UTA & & & $\mathbf{4 0 \%} \mathbf{a} \mathbf{4 3} \%$ \\
\hline
\end{tabular}

Fuente: Elaboración propia basado en Borges, 2019; Borges et al., 2020.

Considerando el cuadro anterior, podemos indicar que existe equidad vertical, entendiendo que ha mayor ingreso mayor es el aporte del tramo mediante impuesto. Por otro lado, podemos considerar que existe una diferencia entre el tramo $7^{\circ}$, que al desaparecer el tramo $8^{\circ}$, este grupo ha obtenido un beneficio de 5 puntos porcentuales, frente a la estructura anterior.

Respecto de los contribuyentes (Cuadro $\mathrm{N}^{\circ} 2$ ), porcentualmente han disminuido, los que más han disminuido son los contribuyentes (2010-2019) tramo 1 (exento) desde $59,1 \%$ a $46,8 \%$ (-9,3\%). Por otro lado, se observa el aumento el tramo 2 desde $20,6 \%$ a $30,1 \%(9,5 \%)$, tramo 3 desde $9,6 \%$ a $11,6 \%$ $(2,0 \%)$ y el tramo $7-8$ desde $1,5 \%$ a $2,4 \%$ $(0,9 \%)$. Indicando que existe un aumento de los ingresos de los sectores con menores recursos, avanzado al tramo 2 y 3 , pero es muy leve el aumento porcentual de contribuyentes en los tramos más altos. Es de- cir, se mantiene casi con pocas variaciones en los demás tramos, excepción el tramo 7 y 8 .

Al analizar el porcentaje de la renta imponible, podemos indicar que la disminución más notoriamente y permanente todos los tramos. La excepción fueron el tramo 2 que aumento de $20,7 \%$ a $23,9 \%(2,2 \%)$ y el tramo $7-8$ de $14,1 \%$ a $22,2 \%(8,1 \%)$. Indicando que la distribución de la riqueza es muy baja por tramo, si consideramos el tramo 1 al 3 concentramos el $88,5 \%$ de los contribuyentes y la renta es $51,6 \%$ de los ingresos renta al 2019. En pocas palabras el $11,5 \%$ de los contribuyentes concentran el $49,4 \%$ de los ingresos rentas. (ver Borges 2021, para profundizar el tema)

Por último, respecto al aporte IGC complementario a aumentado en el tramo $7-8$, desde un $44,2 \%$ a $63,4 \%$ (19,2\% más que en el año 2010).
Finalmente podemos realizar un análisis regional y entender que las rentas que han sido más beneficiadas por la disminución de la tasa de un $40 \%$ a un $35 \%$, se concentran en la Región Metropolitana.

En el gráfico $\mathrm{N}^{\circ} 1$ podemos observar que los aportes de las rentas más altas del país mediante el impuesto a la renta se concentran en la región metropolitana. "Los aportes corresponden al $74,3 \%$ en el 2014 y va en aumento hasta el año 2016, luego se aplicó la disminución a las rentas más altas, produciendo una disminución en el aporte de la Región Metropolitana, esta disminución se debe a que las rentas más altas principales se encuentran en esta región. En conclusión, la medida de la rebaja del $40 \%$ al 35\% generó una desigualdad territorial y por contribuyente, es decir, las regiones entregaron más aportes al Estado." (Borges, 2020 2) Con esto se justifica con mayor razón el aumento de tasa y creación de un nuevo tramo más altos. 
Cuadro $\mathrm{N}^{\circ} 2$

Contribuyentes, Porcentaje de la Renta y Aporte \% del IGC - 2010, 2013, 2016 y 2019.

\begin{tabular}{|c|c|c|c|c|c|c|c|c|c|c|c|c|}
\hline & \multicolumn{3}{|c|}{ IGC 2010} & \multicolumn{3}{|c|}{ IGC 2013} & \multicolumn{3}{|c|}{ IGC 2016} & \multicolumn{3}{|c|}{ IGC 2019} \\
\hline Detalle Tramo & 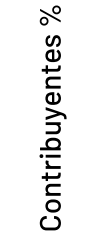 & 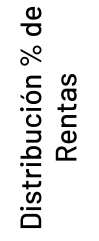 & $\begin{array}{l}0 \\
0 \\
\overline{0} \\
0 \\
00 \\
0 \\
0 \\
\frac{1}{0} \\
\frac{0}{2}\end{array}$ & 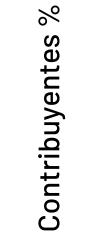 & 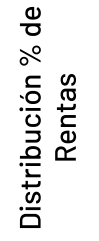 & $\begin{array}{l}0 \\
\text { 음 } \\
0 \\
0 \\
0 \\
0 \\
0 \\
\frac{0}{2} \\
0 \\
0\end{array}$ & 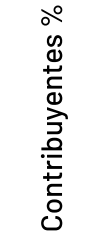 & 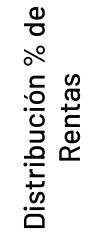 & $\begin{array}{l}0 \\
0 \\
\overline{0} \\
0 \\
0 \\
0 \\
0 \\
0 \\
\frac{1}{0} \\
\frac{0}{0}\end{array}$ & 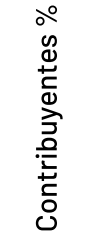 & 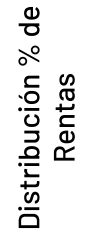 & 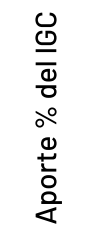 \\
\hline Tamo 1 & $59,1 \%$ & $13,0 \%$ & $0,0 \%$ & $49,3 \%$ & $11,7 \%$ & $0,0 \%$ & $47,1 \%$ & $10,8 \%$ & $0,0 \%$ & $46,8 \%$ & $10,2 \%$ & $0,0 \%$ \\
\hline Tamo 2 & $20,6 \%$ & $20,7 \%$ & $4,3 \%$ & $28,6 \%$ & $24,7 \%$ & $4,3 \%$ & $30,2 \%$ & $24,9 \%$ & $4,0 \%$ & $30,1 \%$ & $23,9 \%$ & $3,6 \%$ \\
\hline Tamo 3 & $9,6 \%$ & $18,4 \%$ & $9,8 \%$ & $10,7 \%$ & $17,8 \%$ & $8,2 \%$ & $11,1 \%$ & $17,5 \%$ & $7,5 \%$ & $11,6 \%$ & $17,5 \%$ & $6,8 \%$ \\
\hline Tamo 4 & $4,7 \%$ & $13,8 \%$ & $11,9 \%$ & $4,9 \%$ & $12,4 \%$ & $9,7 \%$ & $4,8 \%$ & $11,7 \%$ & $8,5 \%$ & $4,8 \%$ & $11,2 \%$ & $7,4 \%$ \\
\hline Tamo 5 & $2,6 \%$ & $10,0 \%$ & $12,4 \%$ & $2,7 \%$ & $9,1 \%$ & $10,8 \%$ & $2,6 \%$ & $8,5 \%$ & $9,4 \%$ & $2,4 \%$ & $7,6 \%$ & $7,6 \%$ \\
\hline Tamo 6 & $1,9 \%$ & $9,9 \%$ & $17,4 \%$ & $2,0 \%$ & $8,9 \%$ & $15,6 \%$ & $2,0 \%$ & $8,4 \%$ & $13,7 \%$ & $1,9 \%$ & $7,5 \%$ & $11,2 \%$ \\
\hline Tamo 7y 8 & $1,5 \%$ & $14,1 \%$ & $44,2 \%$ & $1,8 \%$ & $15,5 \%$ & $51,3 \%$ & $2,2 \%$ & $18,1 \%$ & $56,8 \%$ & $2,4 \%$ & $22,2 \%$ & $63,4 \%$ \\
\hline
\end{tabular}

Fuente: Elaboración propia a partir de la información estadística entregada por el SII.

\section{Gráfico $N^{\circ} 1$}

\section{Aporte del Impuesto Renta de los contribuyentes con}

rentas altas (2014 al 2018). RM - otras Regiones

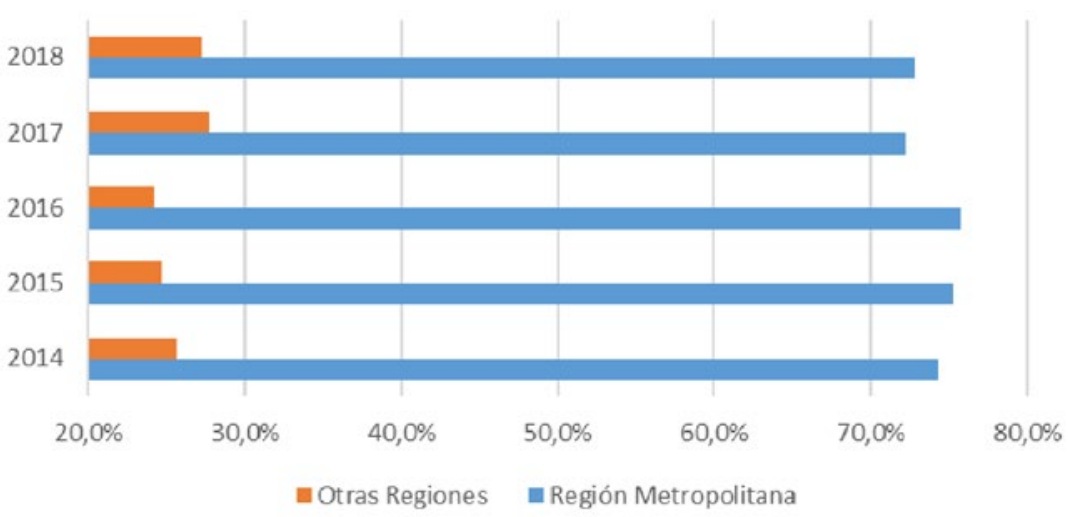

\begin{tabular}{|l|c|c|c|c|c|}
\cline { 2 - 5 } Regiones & $\mathbf{2 0 1 4}$ & $\mathbf{2 0 1 5}$ & $\mathbf{2 0 1 6}$ & $\mathbf{2 0 1 7}$ & $\mathbf{2 0 1 8}$ \\
\hline Región Metropolitana & $74,3 \%$ & $75,3 \%$ & $75,7 \%$ & $72,3 \%$ & $72,8 \%$ \\
\hline Otras Regiones & $25,7 \%$ & $24,7 \%$ & $24,3 \%$ & $27,7 \%$ & $27,2 \%$ \\
\hline
\end{tabular}

Fuente: elaboración propia, en base de la información del SII año 2019. Borges, 20202

\section{Reflexión final}

Al analizar la redistribución de los ingresos tributarios, mediante el análisis de los efectos distributivos totales de los impuestos podemos indicar que se observa que el Impuesto Global Complementario puede recaudar más de las regiones con mayores ingresos, tal como ocurre en la RM. Nuestra propuesta, se enfoca a aumentar el impuesto global tributario, principalmente en el grupo de contribuyentes que fue beneficiado con la política aplicada en el 2014 de disminución de impuestos, que por su parte no fue aumentada en el gobierno actual, que sólo aplicó el $40 \%$ al grupo de rentas superior a los $\mathrm{M} \$ 18.000$. Incorporar un nuevo tramo de contribuyentes de 150 UTA a 310 UTA, que continua con una exención de impuesto, con una tasa del 37,5\% o $40 \%$, con un posible aumento de $40 \%$ a $43 \%$ en el tramo de 310 UTA. 


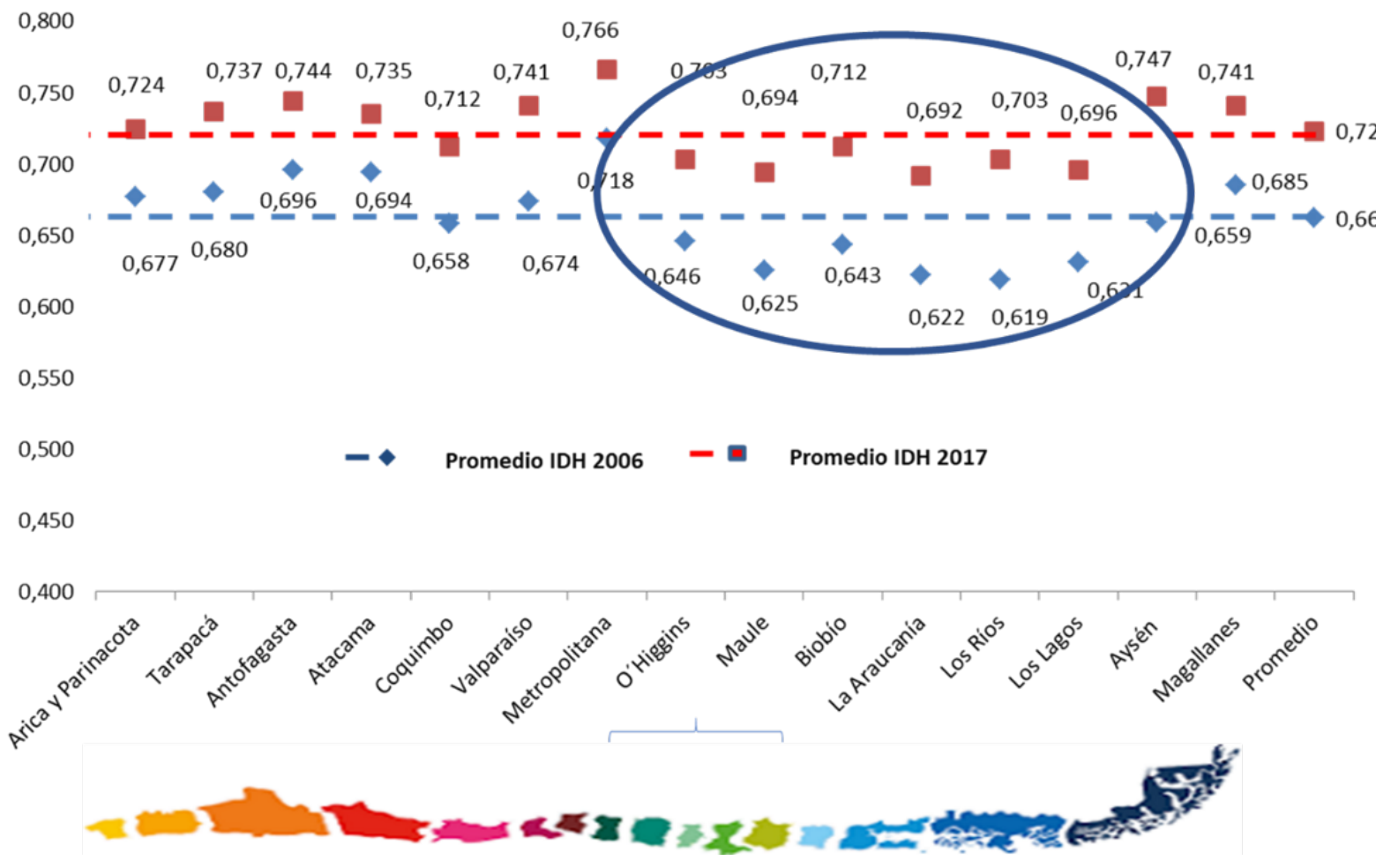

\begin{tabular}{|c|c|c|c|c|c|c|c|c|c|c|c|c|c|c|c|c|}
\hline Regiones & 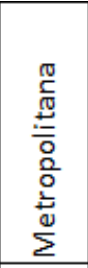 & 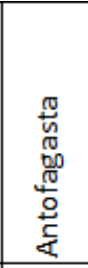 & 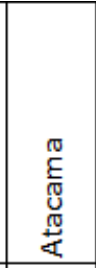 & 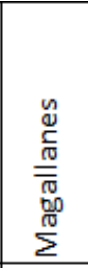 & 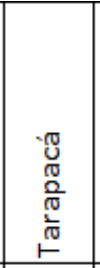 & 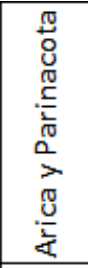 & $\begin{array}{l}\frac{0}{2} \\
\frac{n}{\pi} \\
\frac{2}{\pi} \\
\frac{2}{\pi} \\
> \\
>\end{array}$ & 离 & 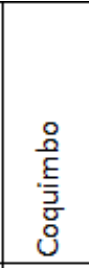 & 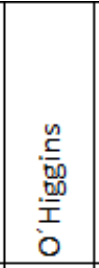 & $\frac{\circ}{\frac{0}{0}}$ & 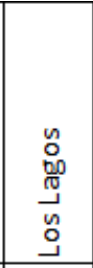 & $\frac{\frac{\omega}{J}}{\sum}$ & 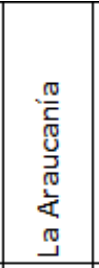 & 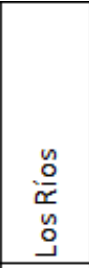 & Promedio \\
\hline IDH 2006 & 0,718 & 0,696 & 0,694 & 0,685 & 0,680 & 0,677 & 0,674 & 0,659 & 0,658 & 0,646 & 0,643 & 0,631 & 0,625 & 0,622 & 0,619 & 0,662 \\
\hline $\begin{array}{c}\text { Posición en } \\
2006\end{array}$ & 1 & 2 & 3 & 4 & 5 & 6 & 7 & 8 & 9 & 10 & 11 & 12 & 13 & 14 & 15 & \\
\hline IDH 2017 & 0,766 & 0,744 & 0,735 & 0,741 & 0,737 & 0,724 & 0,741 & 0,747 & 0,712 & 0,703 & 0,712 & 0,696 & 0,694 & 0,692 & 0,703 & 0,723 \\
\hline $\begin{array}{c}\text { Posición en } \\
2017\end{array}$ & 1 & 3 & 7 & 5 & 6 & 8 & 4 & 2 & 9 & 11 & 10 & 13 & 14 & 15 & 12 & \\
\hline
\end{tabular}

Fuente: PNUD (2018) y Borges (2019).

\section{REFERENCIAS}

- $\quad$ BORGES, H. 2019. Discusiones tributarias y realidad regional. Observatorio Económico 136: 6-7.

- $\quad$ BORGES, H. 2020 1. Relación de los Impuestos Personales con el Desarrollo Humano Regional: Caso de Chile. CAPIC REVIEW, 18, 1-15.

- BORGES QUINTANILLA, H. 2020 2. Chile en busca de justicia tributaria: Propuestas impositivas en un momento de cambio estructural. Observatorio Económico, 8-9.

- BORGES, H. (2021). Radiografía de los ingresos tributarios de los impuestos personales (2010 al 2019). Observatorio Económico, (156), 2-5. https://doi. org/10.11565/oe.vi156.416

- $\quad$ DE ZUAZU, M. 2012. Elementos principales de la cultura fiscal en América Latina. Documentos de trabajo. IELAT, Instituto Universitario de Investigación en Estudios Latinoamericanos 42: 40-59.

- GARRETÓN, M., JOIGNANT, A., SOMMA, N. Y CAMPOS, T. 2018. Informe Anual Observatorio de Conflictos. Nota COES de Política Pública, 17.

- JORRATT, M Y MARTNER, R. 2020, noviembre. JUSTICIA FISCAL PARA CHILE, El impuesto a la riqueza y otras reformas tributarias progresivas. Justicia-fiscal-paraChile.pdf (aneiich.cl).

- $\quad$ PROGRAMA DE NACIONES UNIDAS PARA EL DESARROLLO (PNUD). 2018. Desigualdad regional en Chile. Ingresos, salud y educación en perspectiva territorial. www.desiguales.org.

- $\quad$ Revenue Statistics in Latin America and the Caribbean 2020 | en | OECD. 